An NAS report questions whether current tolerance-setting procedures adequately protect children as well as adults from unhealthy exposures to pesticide residues.

Tolerances, the legal limits of pesticide residues in food, should be health-based standards with adequate safety margins for all consumers, including infants and children. The current pesticide regulatory system does not ensure this. Tolerances are set to reflect "good agricultural practice." If health-based standards are to be developed, regulators must collect more adequate toxicological data, and data on children's food consumption patterns. While basic changes are needed in the current regulatory system, consuming a diet rich in fruits and vegetables confers great health benefits. The recommendations of the NAS committee sought to make our very good food supply even better.

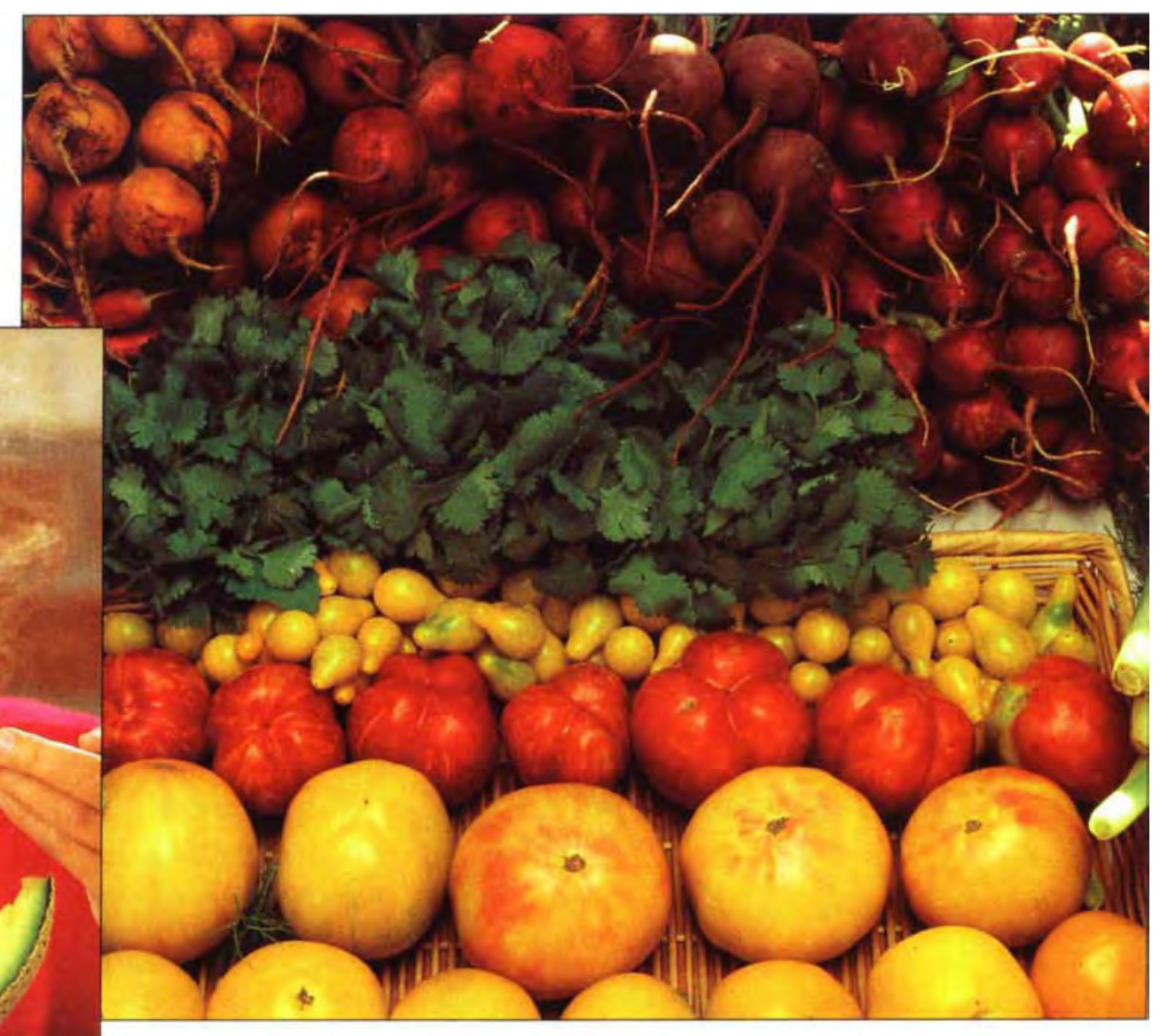

\title{
One size does not fit all
}

\author{
Some thoughts on pesticides in the diets of \\ infants and children
}

Richard J. Jackson

A $\mathrm{n}$ abundant variety of healthy and safe foods is essential to public health. Concern that the current pesticide regulations do not protect sensitive members of the population - especially infants and children prompted Congress to order the $\mathrm{Na}$ tional Academy of Sciences (NAS) report Pesticides in the Diets of Infants and Children, released in June 1993. The report was prepared by a committee that included pediatricians, agricultural chemists, toxicologists and representatives from public health, government, academe and industry.
Pediatrician and epidemiologist $\mathrm{Ri}$ chard Jackson was one of 14 members of the National Academy of Sciences committee that released Pesticides in the Diets of Infants and Children in June 1993 after nearly 5 years of preparation. He is Chief, Division of Communicable Disease Control, California Department of Health Services, and holds a UCSF appointment in the Division of Environmental and Occupational Medicine.

-Editor 


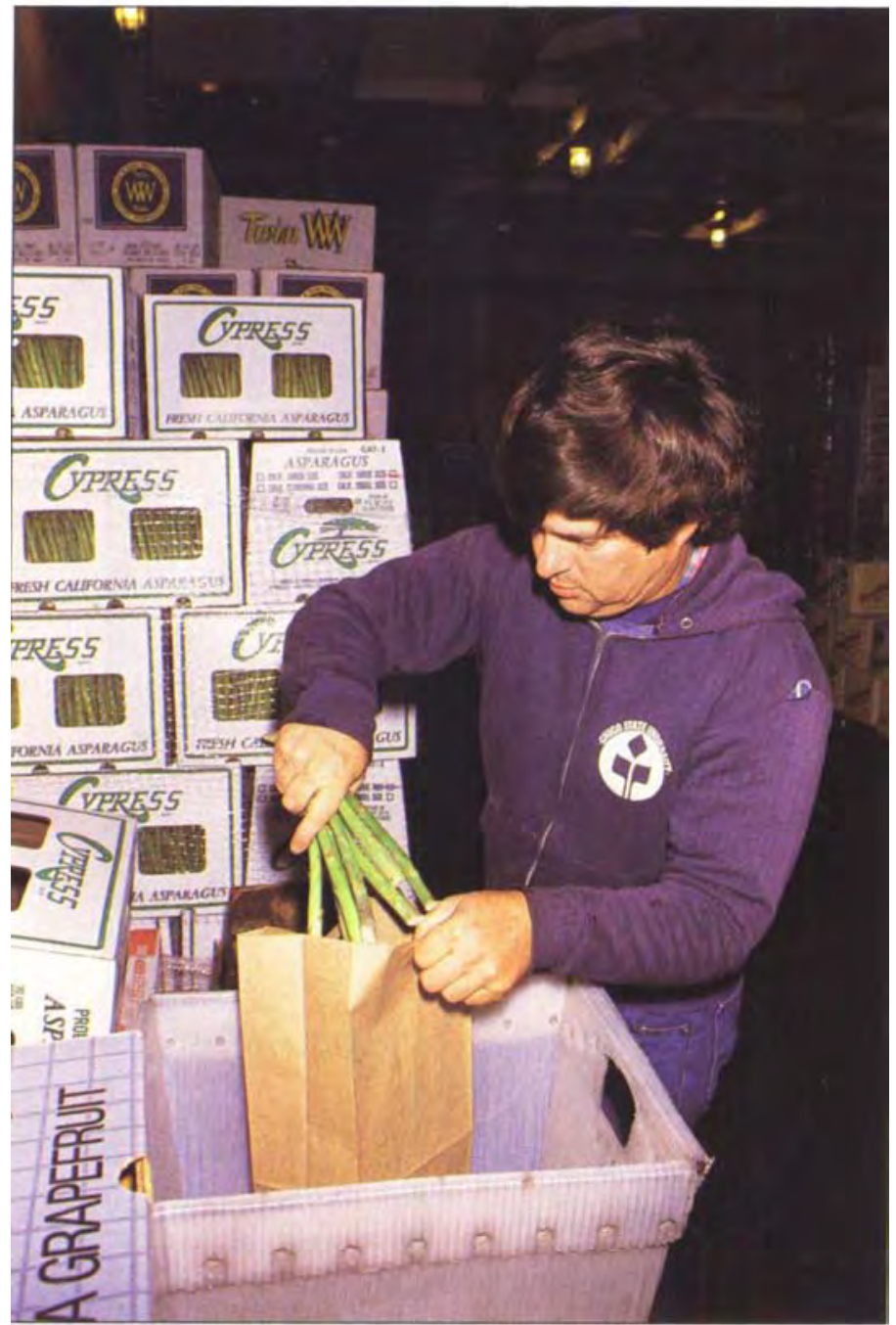

CDFA technician samples asparagus for pesticide residues. Most foods do not have large detectable residues; dietary hazards for the general population are low.

The core message of the report is that "one size does not fit all" when it comes to pesticide regulation; infants and children are not "little adults" and the regulatory system needs to set limits to protect them. Currently it does not. Tolerances, the legal limits for residues in food, are not set on a health basis, not even for adults. Only limited data on post-gestational toxicity are available in the pesticide registration data base. Because developing animals can be more sensitive than mature animals to carcinogens and other toxicants - although in some cases they are more resistant - additional safety factors for early childhood exposures should be required unless there are data to show this is unnecessary.
Also, because of small sample sizes, the data bases are inadequate on children's food consumption patterns nationally and regionally, as well as on the level of actual residues in food. Finally, children are exposed to multiple pesticides that have common toxicologic effects, and they are exposed in ways other than by eating, for example by playing on contaminated surfaces. The regulatory system does not account for this.

\section{Health tolerances}

Pesticide tolerances have traditionally been set to reflect "good agricultural practice," that is, if a grower complies with the pesticide label restrictions, the residues on the crop should fall below the tolerance limits. While tolerances help enforce pesticide regulatory compliance, in many cases they do not adequately protect all members of the population. A central conclusion of the NAS report was that tolerances should be set to protect health and should afford adequate margins of safety to all consumers.

Pesticide food tolerances are the benchmark of the pesticide regulatory system. These levels determine the acceptability of food for commerce and theoretically for consumption. They are the basis for large and expensive food residue testing programs and are used to justify the assertion: "the food supply is safe, all the residues are below tolerances." The maximum allowable amount of pesticide residues in food, the theoretical maximum residue concentration (TMRC), is estimated by assuming all the food we eat in a day contains the legal limit of pesticide residues. This amount in many cases greatly exceeds levels that would be permitted on a health basis, the acceptable daily intake (ADI). (For further details, see pp. 19.) Setting a healthbased limit for all legal residues would result in more stringent tolerances with more focus on essential uses.

While the committee emphasized the need for health-based tolerances to protect all members of the population, it recognized that most of the time personal hazards from dietary pesticide residues are low. In reality, most foods do not have large detectable residues. A diet rich in fruits and vegetables and low in fat, salt and refined sugars, has well-documented disease prevention benefits. The committee emphasized that these are excellent foods, and child-protective regulation would make them even better.

The system of tolerances has brought benefits. It is useful to ensure pesticide label compliance and to protect growers who adhere to the labels from facing condemnation of a crop that represents great financial and personal investment. It is also welldocumented that when growers fail to adhere to label requirements, the results can be disastrous. Dramatic episodes have occurred where illegal residues have caused severe illness: in 1985, residues of the extremely toxic insecticide aldicarb sickened 1,000 individuals in the Western U.S. and were associated with two fetal deaths. Illegal levels of the organochlorine endrin in a snack food caused convulsions and loss of driving privileges in healthy young men in Orange County, California, in 1988.

However, the committee could identify no recent cases where legal use of pesticides is documented to have caused human illness from food consumption. (This is in contrast to numerous cases of pesticide-related illnesses in the workplace, where exposures are far greater. See sidebar, p. 17.) While this is reassuring, the committee recognized how extremely difficult it is for a parent or physician to discern that a child's symptoms of muscle twitching, vision disturbance and diarrhea are due to a pesticide in one of a dozen foods eaten in a day. Such subtle and nonspecific symptoms could be occurring without definable cause. 


\section{Children are not "little adults" - NAS report}

$\mathrm{M}$ ajor conclusions of the NAS Report Pesticides in the Diets of Infants and Children include:

Children are not "little adults." Their surface-to-volume ratio is three times higher; they eat fewer kinds of foods and consume more of certain foods per unit of body weight. They also drink more water both alone and mixed with other foods. As children grow, their tissues increase rapidly in cell number and size, and pass through several stages of development. Children's metabolic rates are higher, and they are subject to different levels of enzyme induction and action.

Toxicity testing of immature animals. Risk estimates, tolerances and other food safety determinations should be based on testing that includes immature animals, so that potential sensitivities of infants and children can be evaluated. Some data suggest that children may be more sensitive than adults to some pesticides, while being equally or less sensitive to others.

Better laboratory tests must be developed to measure effects on immature animals. In particular, tests should be developed to assess effects

Children eat fewer kinds of foods and more of certain foods per unit of body weight.

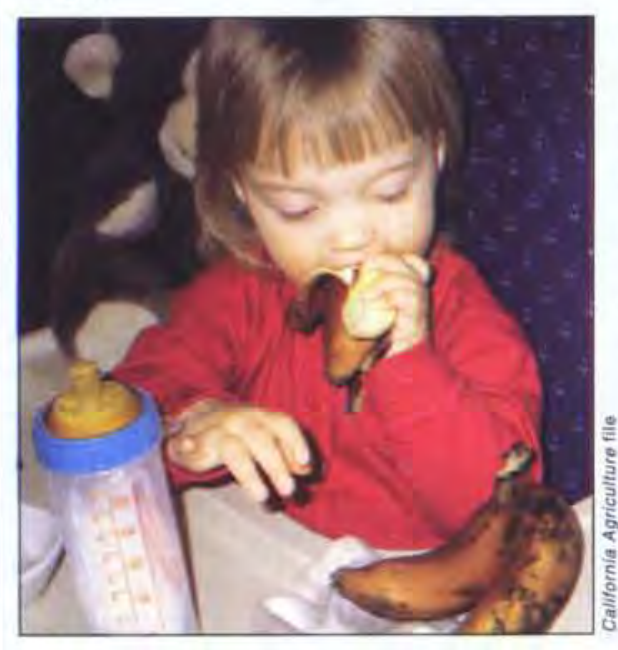

on the immune, neurologic, and reproductive systems.

Uncertainty factors. Use of an additional "uncertainty factor" of up to 10 -fold should be used when there is evidence of postnatal toxicity, or when data relevant to children are incomplete. This precaution - resulting in a 1,000 -fold safety factor - is already taken in cases of prenatal toxicity.

(Currently, if animal tests show no adverse effects at a certain exposure level, the reference dose, or acceptable daily intake, is obtained by dividing that level by 100 - to account for differences between humans and animals and variations among humans.)

Risk distributions. Risk assessment currently takes a "one-size-fits-all" approach to estimating pesticide risk. Each pesticide risk estimate is a single number representing the average risk of an entire population. Instead, assessments should use "risk distributions" combining data that reflect variations in food intake and pesticide residues. (For instance, distributions would reflect food intake variations according to factors such as age, geographic region and ethnic group).

Food consumption data. Because sufficient data are not currently available to permit wide application of these methods, food consumption data should be collected with age-related, geographic, ethnic and cultural differences in mind. Food consumption surveys should be conducted at seven age levels - for every one-year interval up to age 5 , for young children ages 5 to 10 , and for older children ages 11 to 18 .

Multiple exposures. All exposures to pesticides should be considered in determining dietary tolerances. Nondietary sources of possible concern include drinking water, air, soil, lawns, pets and indoor surfaces. Due to a child's constant tasting, touching and manipulating of the world at hand, most of these sources are more important for children than for adults.
Additive effects. Estimates of total dietary exposure should be refined to consider possible additive effects for pesticides having common mechanisms of action, as well as synergistic effects of exposure to multiple pesticides having common toxicological effects.

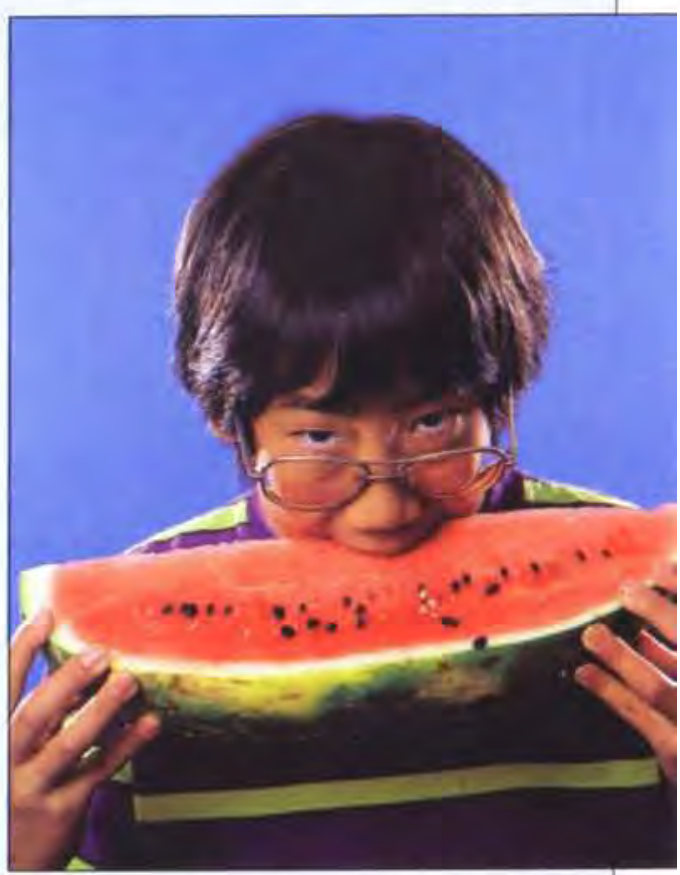

Children may be more sensitive than adults to some pesticides.

Pesticide residue data. Uniform analytical methods and reporting procedures should be used by all laboratories that measure pesticide residues so that data from different laboratories can be reliably compared. The committee also recommended establishment of a computerized data base for collating measurements from different laboratories.

This summary was derived from the NAS report Pesticides in the Diets of Infants and Children and the article "Report Elicits Promises to Improve Pesticide Rules," published in the National Research Council's magazine NewsReport, Summer 1993. -Editor 


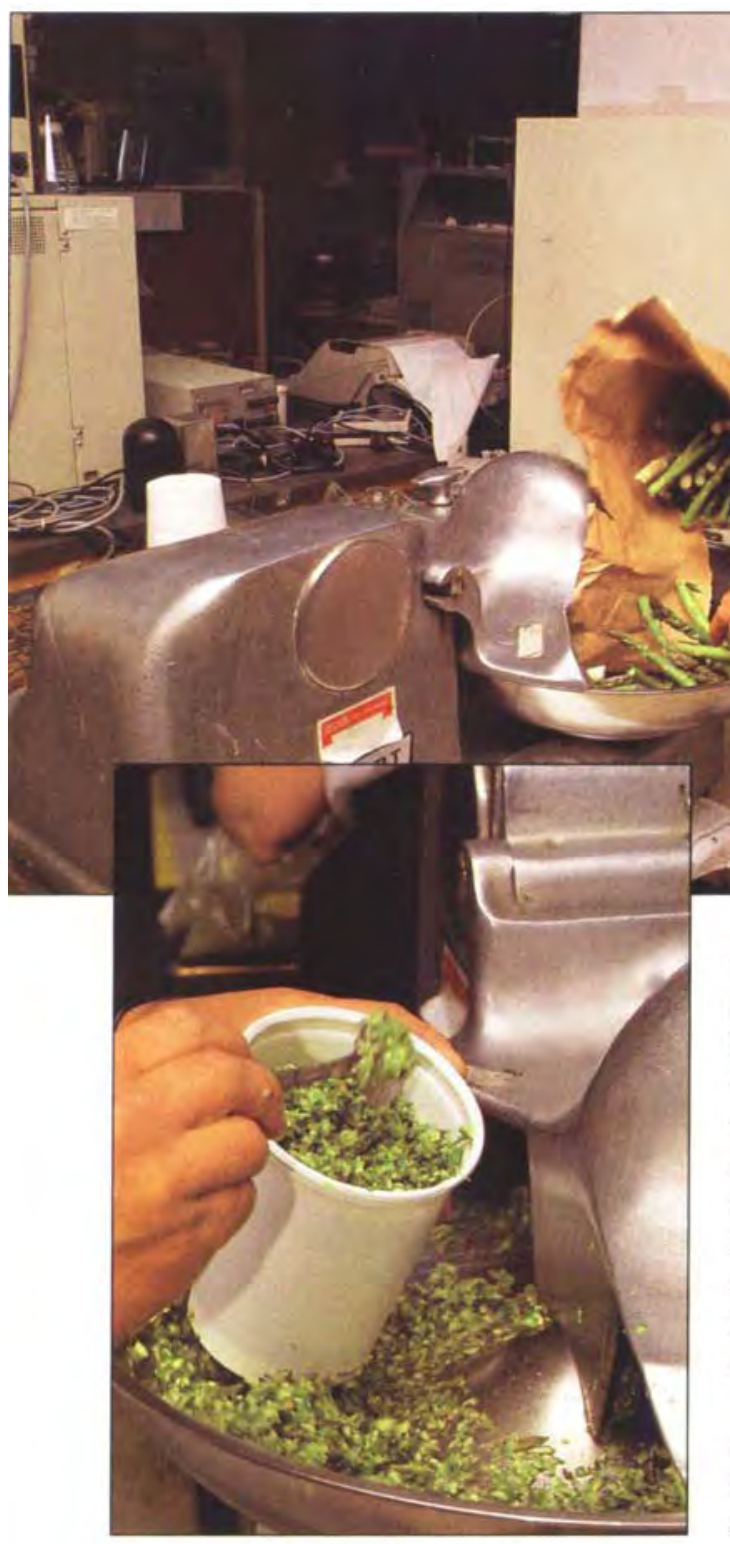

Asparagus samples are ground for residue testing. The NAS report recommended that pesticide residue laboratories nationwide adopt uniform methods of analysis and reporting.

\section{The case of aldicarb}

In the case of aldicarb (Temik), an insecticide with very high acute toxicity, legal limits in potatoes and bananas were set at excessively high levels. For example, an infant eating half a banana at tolerance would be exposed to 6.5 times the ADI. For many years, sampling of aldicarb was done using large batches of food (100 pounds). In the 1980 s it was discovered that the toxic residue that appeared in food was not the parent compound aldicarb, but the degradation product, aldicarb sulfoxide, which is also extremely toxic and very persistent. It was also discovered that

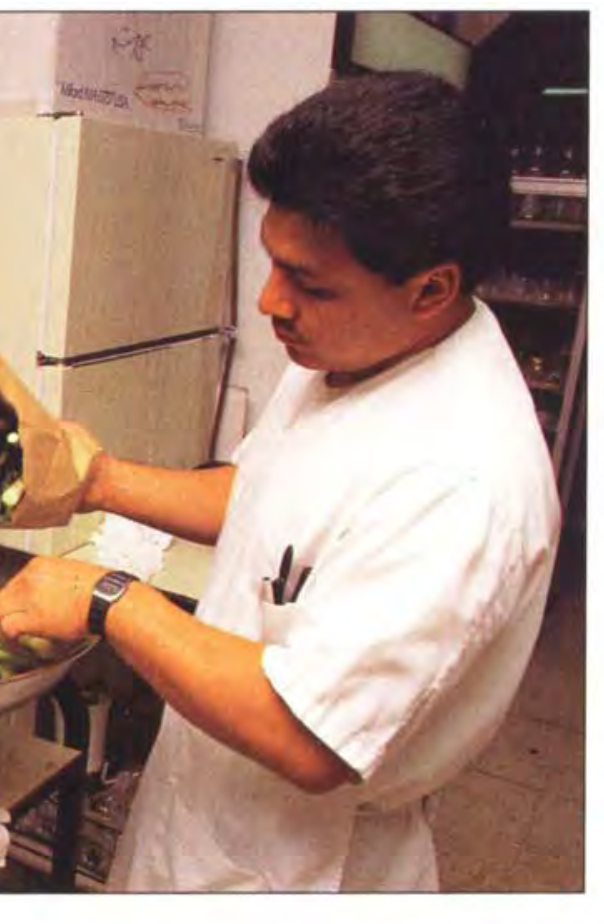

while the average aldicarb level in 100 pounds of potatoes might be relatively low, individual potatoes might contain high levels. One potato was identified with enough aldicarb sulfoxide to give a child consuming it a dose 465 times the ADI and within a margin of 10 from a lethal dose (LD50). Aldicarb is no longer permitted for use on potatoes and bananas, although citrus use continues.

The NAS report documents that toxicologic data bases, food consumption surveys, and pesticide residue analysis programs are, in many cases,

inadequate. It also documents that regulators have been slow to deal with chemicals such as daminozide that may present child health risks. Daminozide (Alar), a plant growth regulator, was registered for food crops in 1968. The manufacturer, Uniroyal, did not perform the required cancer studies in rodents until 1985, after being ordered to do so. Alar was used on apples. Studies done by other groups had shown daminozide's degradation product, UDMH (unsymmetrical dimethyl hydrazine), to be an animal carcinogen, and it was declared to be a potential human carcinogen by both EPA and the International Agency for Research on Cancer. Because young children consume large amounts of apples and apple products, many pediatricians and parents were concerned and urged EPA to suspend use of Alar on products consumed by children.
However, EPA regulators were slow to respond. They first consulted with an advisory committee that contained no pediatric or public health expertise and elected to allow the manufacturer 5 more years to do the definitive study on daminozide's cancer potential. Uniroyal's own study showed that male mice fed UDMH at 40 parts per million had a $60 \%$ cancer rate. Daminozide is no longer permitted for use on food crops. (EPA canceled all corresponding tolerances when Uniroyal voluntarily removed Alar from food crop use in 1989.) While the EPA's final disposition of Alar was appropriate, its delay in action was not. Because of the evolving nature of science and toxicology, it is essential that regulators be equipped to respond in a timely fashion to new evidence of adverse health effects even if a pesticide is registered and on the market.

The toxicology data base that is needed to ensure safety, including safety to children, is always evolving. Developing the information to get a pesticide licensed for food use is costly in time and resources. The National Academy of Sciences in its 1984 study, Toxicity Testing: Strategies to Determine Needs and Priorities, documented that the animal testing data base was profoundly inadequate for more than two-thirds of pesticides. This ultimately led to the passage in California of Senator Nicholas Petris's Birth Defects Prevention Act of 1984, a bill sponsored by health groups and opposed by the agricultural chemical industry and the California Department of Food and Agriculture.

This bill, sometimes known as SB 950, signed into law by Governor Deukmejian, led to the filling of data gaps and has had profound effects on California pesticide use: some have been removed and others have had their re-entry and preharvest intervals increased. One result was that closer scrutiny was applied to all hazards associated with pesticides, particularly the hazards to those most highly exposed, pesticide applicators and farmworkers. While margins of safety conferred upon the consumer may be inadequate, the hazard for these 


\section{Emerging health concerns about pesticide residues}

\author{
William Pease
}

F or more than 30 years, most public ticide residues in food has focused on whether life-long exposure to some chemicals in the diet could increase the incidence of cancer. The Delaney Clause, a 1958 law banning the use of animal carcinogens as food additives, remains at the center of the current Congressional debate over pesticide reform. There is growing interest, however, in examining whether pesticide exposures could have other adverse effects on human health. Public concern about diseases other than cancer has been stimulated by the results obtained from more thorough toxicity testing of pesticides. As scientific understanding of the full range of pesticide toxicity has improved, a new question is being asked in policy debates: Can exposure to pesticide residues in our diet harm our reproductive, neurological or immune systems?

Assessing these potential risks involves many of the same scientific uncertainties that plague current efforts to estimate cancer risks. It is clear from the results of toxicological studies on laboratory animals that many pesticides in widespread use have the capacity to damage important physiological systems. There is also epidemiological evidence that workers with relatively high levels of pesticide exposure can suffer from a variety of acute and chronic health effects.

Some pesticides are reproductive toxicants, capable of damaging the human reproductive system. The normal sperm count of workers formulating DBCP (dibromochloropropane), for example, was lowered substantially because this nematocide is toxic to critical germinal cells in the male testes. Highly exposed workers became infertile and were unable to father children. Other pesticides (for example, organochlorine insecticides like endosulfan) mimic the activity of hu- man hormones and may affect female reproductive capacity. Toxicological evidence indicates that many pesticides can also cause birth defects: Of some 200 pesticides tested to date, nearly half are reported to induce birth defects in experimental animals.

Some pesticides can damage the immune system, triggering hypersusceptiblity to chemical exposures or hindering an organism's ability to successfully fight off infections. Occupational exposures to captan, for example, can induce contact hypersensitivity, a type of dermatitis. Toxicological studies have demonstrated that other pesticides can damage the immune system. Pesticides such as carbofuran and methyl parathion, for example, reduce an experimental animal's capacity to resist bacterial infections.

Entire classes of pesticides, like the organophosphate and carbamate insecticides, target enzymes that are essential to the functioning of a healthy nervous system. Farmworker exposures to mevinphos and methomyl, for example, have resulted in several mass poisoning incidents. Workers experience both physical and neurological symptoms, including nausea, breathing difficulties, irritability and confusion. Epidemiological studies indicate there can also be long-term damage (such as memory disturbances and deficits in intelligence and motor functioning) as a result of repeated acute exposures to neurotoxic pesticides.

Whether these types of adverse effects are occurring in humans as a result of dietary exposures to pesticide residues is largely unknown. Such endpoints often involve nonspecific symptoms. It is also difficult to characterize the pesticide exposures people experience. There is a complex mixture of many different pesticides in normal diets, as well as a number of other potential routes of exposure (for example, home consumer product

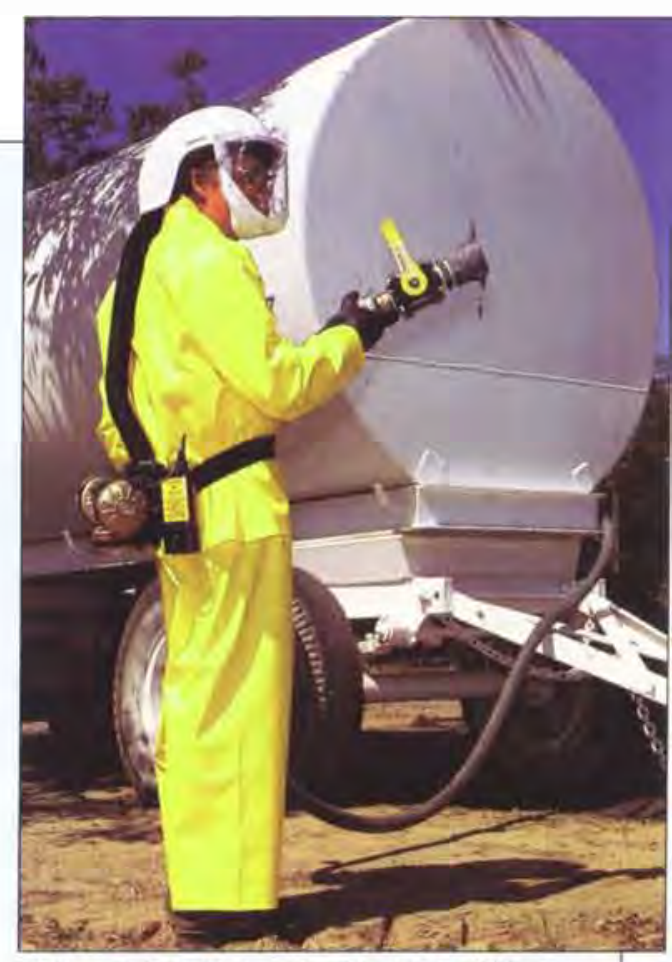

This pesticide applicator is outfitted with a respirator and protective clothing. Occupational exposures to pesticides are far greater than those from food consumption.

use). This has made it virtually impossible to conduct epidemiological studies of the noncancer effects of lowlevel dietary exposures.

Current scientific efforts compare estimated dietary intakes with pesticide dose levels known to cause adverse effects. The recent NAS report on pesticides in children's diets, for example, examined whether the cumulative intake of different pesticides that inhibit cholinesterase could have adverse neurological consequences. The report found that while the vast majority of children do not consume sufficient quantities of pesticide residues to raise any health concerns, about $1 \%$ of children on any given day are receiving pesticide doses that exceed EPA's acceptable exposure level. This finding has not resolved whether serious noncancer effects can be associated with food residues. Rather, it represents a new phase in the debate over food safety, as regulators begin to focus on reducing cumulative effects potentially associated with the variety of pesticide residues in the food supply.

W. Pease is Toxicologist, Environmental Health Sciences, School of Public Health, UC Berkeley. 


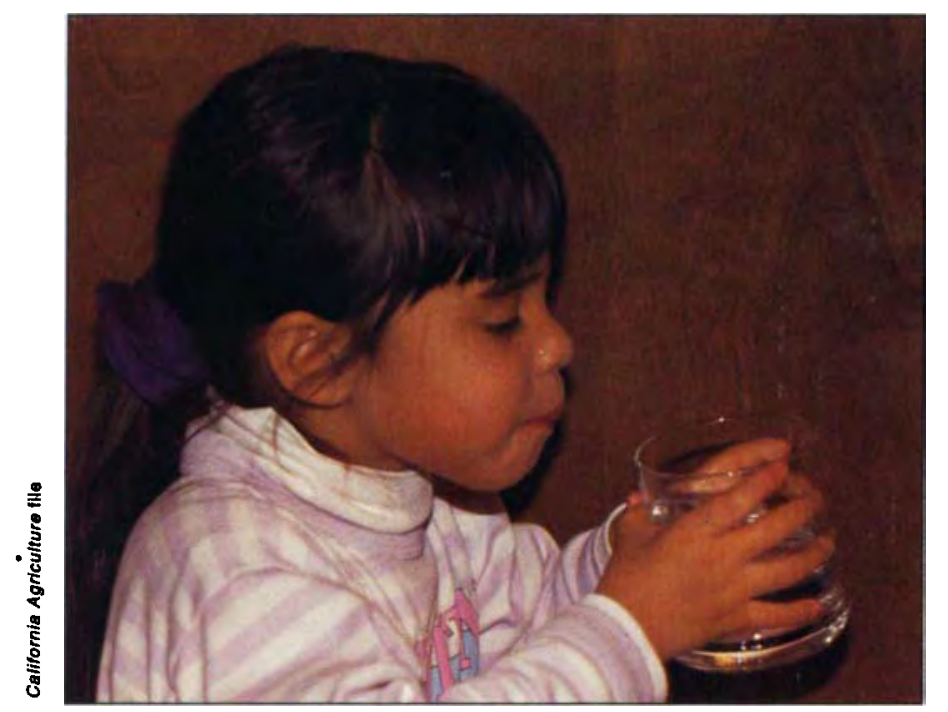

Nonfood exposures, including drinking water, should be considered when setting tolerances for food eaten by chlldren.

groups is far higher, and more worker protection efforts than food tolerance changes have resulted from the Act.

\section{Children's data gaps}

While data gaps have been filled over the last 10 years, the array of needed data increases. In the medical research area we have learned much more about the hazards of toxic agents to the immune and nervous systems, and to developing organisms. Yet there are only limited data on toxicity to infants and children in the pesticide registration data base. Pesticide regulatory requirements have not kept pace, in part because research discoveries and tools need to be well validated before they can be required of a manufacturer seeking to document safety. In toxicologic studies, it is very difficult to find animal counterparts to, for example, the human 2-year-old. Human development takes much longer than that of any test animal species, with far wider "windows of vulnerability." We may well end up needing to use computer models for many developmental exposures and to apply additional safety factors so we can ensure that children are protected.

Children eat a smaller variety of foods and consume more food and water on a body-weight basis than adults. A toddler who weighs oneseventh as much as an adult may con- sume 1,000 calories per day, yet many active adults get by on only two to three times that amount. To offer toddlers the same degree of protection as adults, the allowable residue levels permitted for children need to be roughly three times more stringent than those allowable for adults.

An adult has seven times the weight but only three times the surface area of a toddler. The child's larger surface-to-volume ratio coupled with its "occupation" of touching, tasting and manipulating its environment puts it at greater risk from pesticide exposure on surfaces such as play structures, decks, rugs and lawns. This reality is not incorporated into pesticide decision-making. The allowable levels for pesticides in food are set without recognizing that children are exposed to pesticides from treated surfaces. No margin is left over for nonfood exposures, including even drinking water exposures.

Pesticides are present in drinking water - extensively in California groundwater - as well as in air, in workplaces and on surfaces. When EPA sets allowable levels for pesticides in drinking water, it does not allow exposure to an amount that would exceed an ADI, in fact it tries to limit exposure to only $20 \%$ of the ADI, assuming correctly that there will be routes of exposure other than water. In contrast, when setting food levels, the regulators use formulas that assume that only food exposures occur.

\section{"Tower of Babel"}

One reason the NAS report took so long in preparation, almost 5 years, was that the committee expected the gathering of pesticide residue data to be relatively simple. It was not. We expected that computerized data bases listing residue levels, with information as to the laboratory method used, the level of quantification, records of which chemicals were actually used, and quality assurance data would be available. They were not. The many laboratories - federal, state, or private - involved in food testing use many different analytical methods with differing sensitivities, different data base software, and no record-keeping as to whether pesticides were actually used on the food. Some laboratories did not even keep a record of negative results, recording only the "detects." This "tower of Babel" could be rectified with good federal-state partnership efforts without great cost.

Finally, pesticides that have similar toxicologic effects need to be regulated as a group. This is not now done. For example, new pesticides are registered without recognition that there may be other chemicals that are used on foods at the same time and that additive toxicologic effects could result. Using complex computer simulations, the NAS committee used data from actual food consumption and pesticide residue surveys to estimate that, with just five organophosphates on only eight foods, $1.3 \%$ of children would be exceeding the acceptable daily intake for a reference organophosphate, and about a tenth of those would be in a range where symptoms could occur. While this sounds like a relatively low frequency, when rare events occur among tens of millions of children, a large number could be ill, though no clinician would be able to make the diagnosis.

Pesticide residue levels should be set at health protective levels for children. The health of all would be better protected, and not only from exposure to food residues. Farmworkers would be exposed to less residue on the crops with which they work, and each pesticide use would be more closely examined for need and benefits. Less pesticides in the air, water and food would benefit our children, and theirs.

R. J. Jackson is Assistant Clinical Professor, Department of Medicine, Division of Occupational and Environmental Medicine, UC San Francisco. 